6. Kumar P, Angst DB, Taxy J, et al. Neonatal autopsies: a 10year experience. Arch Pediatr Adolesc Med 2000;154(1):38-42.

7. Swinton $\mathrm{CH}$, Weiner J, Okah FA. The neonatal autopsy: can it be revived? Am J Perinatol 2013;30(9):739-44.

8. Hickey L, Murphy A, Devaney D, etal. The value of neonatal autopsy. Neonatology 2012;101(1):68-73.

9. Rose C, Evans M, Tooley J. Falling rates of perinatal postmortem examination: are we to blame? Arch Dis Child Fetal Neonatal Ed 2006;91(6):F465.

10. Burton JL, Underwood J. Clinical, educational, and epidemiological value of autopsy. Lancet 2007; 369(9571):1471-80.
11. Dhar V, Perlman M, Vilela MI, et al. Autopsy in a neonatal intensive care unit: utilization patterns and associations of clinicopathologic discordances. J Pediatr 1998;132(1):75-9.

12. Faye-Petersen OM, Guinn DA, Wenstrom KD. Value of perinatal autopsy. Obstet Gynecol 1999;94(6):915-20.

13. Custer JW, Winters BD, Goode V, et al. Diagnostic errors in the Pediatrics and Neonatal ICU: A Systematic Review. Pediatr Crit Care Med 2015;16(1):29-36.

14. Schnitzler E. Volver a la autopsia o seguir ignorando la verdad. Arch Argent Pediatr 2003;101(3):163-4.

15. EscardóF. Sentidoético dela autopsia. Boletín de la IICátedra de Pediatría 1964;2.

\title{
Serum prolactin levels in atopic dermatitis and the relationship with disease severity
}

\author{
Burcu Tuğrul Ayanoğlu, M.D. ${ }^{a}$, Özgül Muştu Koryürek, M.D. ${ }^{a}$, Songül Yıldırım Başkara, M.D. ${ }^{b}$
}

\begin{abstract}
Background. Prolactin performs as a neuroendocrine modulator of skin epithelial cell proliferation and the skin immune system. Objective. The aim was to assess the serum prolactin levels in patients with atopic dermatitis and the relationship with disease severity.

Methods. The study was performed on 46 patients with atopic dermatitis and 100 healthy controls aged between 0.5 years and 19.5 years. The diagnosis of atopic dermatitis was based on clinical findings and the severity of the disease was documented. Venous blood sampling was performed in order to measure prolactin levels.

Results. Prolactin levels in atopic dermatitis were not different from controls and there was no relationship between the severity of atopic dermatitis and serum prolactin levels. Prolactin may not have a role in the pathogenesis of atopic dermatitis. Further studies with larger sample sizes and measurement of prolactin levels in the skin may help to understand the role of prolactin in the pathogenesis of atopic dermatitis.

Keywords: Atopic dermatitis, prolactin, SCORAD index, child.
\end{abstract}

http:/ / dx.doi.org/10.5546/ aap.2017.eng.493

To cite: Tuğrul Ayanoğlu B, Muştu Koryürek Ö, Yıldırım Başkara S. Serum prolactin levels in atopic dermatitis and the relationship with disease severity. Argent Pediatr 2017;115(5):493-496.

a. Aksaray University, Aksaray Training and Research Hospital, Department of Dermatology and Venereology. Aksaray, Turkey.

b. Aksaray University, Aksaray Training and Research Hospital, Department of Pediatrics. Aksaray, Turkey.

E-mail address:

Burcu Tuğrul Ayanoğlu, M.D.: burcutugrul@yahoo.com

Funding: None.

Conflict of interest: None.

Received: 12-20-2016

Accepted: 5-16-2017

\section{INTRODUCTION}

Atopic dermatitis (AD) is a chronically relapsing inflammatory skin disease associated with complex interactions among susceptibility genes, the host's environment, defects in skin barrier function and systemic and local immunologic responses. The disease is characterized by eczematous skin lesions and intense pruritus that often occur in early childhood but can also start or persist in adulthood. ${ }^{1}$ Scoring systems have been developed for use in trials.

Prolactin (PRL) is a polypeptidic neuropeptide produced by lactotroph cells in the anterior pituitary gland and is well known for its lactogenic and mammotrophic effects. It has been hypothesized that forming a 'prolactincircuit' between the central nervous system and the skin, prolactin performs as a neuroendocrine modulator of skin epithelial cell proliferation and of the skin immune system. PRL plays an important role in the immune reactions and exerts a proliferative effect on human keratinocytes by binding specific sites. ${ }^{4}$ Previous studies have assessed the association of PRL levels with psoriasis as a hyperproliferative cutaneous disease of multifactorial etiology; however, the results seem contradictory. Epidermal hyperproliferation that is observed in both psoriasis and atopic dermatitis and its underlying mechanisms are not completely understood by now. ${ }^{8}$

Only some rare information is available on the 
PRL levels in children with AD. In the present study, we measured serum PRL levels in patients with $\mathrm{AD}$ and studied the correlation between clinical disease activity and PRL.

\section{MATERIALS AND METHODS \\ Patient selection}

This prospective analytic case-control study was conducted at the Aksaray Training and Research Hospital, between March 2014 and January 2015. The study protocol was approved by the local ethics committee, written informed consent was obtained from each patient and their parents. We studied 46 atopic patients in exacerbation periods and 100 sex and age matched patients who were admitted to the Dermatology and Pediatrics Outpatient Clinics other than atopic dermatitis at the same time interval. The study group was selected from the patients that would have already given routine blood tests. Exclusion criterias were defined as refusal to participate in the study, having medical conditions or using drugs which could alter the levels of prolactin such as thyroid disorders, renal and/or hepatic failure, having pregnancy and other autoimmune or inflammatory disorders that could coexist with atopic dermatitis. The diagnosis of atopic dermatitis was based on clinical findings and history of the disease. All the patients and the parents filled out the questionnaire which included their demographic data and medical history (age, sex, duration of the disease, number of relapses). Severity of disease was documented by using the SCORing Atopic Dermatitis (SCORAD) index which is the best validated scoring system in AD. In SCORAD index, to measure the extent of $\mathrm{AD}$, the rule of nines is applied on a front/back drawing of the patient's inflammatory lesions. The extent can be graded from 0 to 100 . The intensity part of the SCORAD consists of 6 items: erythema, edema/ papulation, excoriations, lichenification, oozing/crusts and dryness. Each item can be graded on a scale from 0 to 3 . The subjective items include daily pruritus and sleeplessness. The SCORAD Index formula is: $A / 5+7 B / 2+$ $C$. In this formula $\mathrm{A}$ is defined as the extent (0$100), B$ is defined as the intensity (0-18) and C is defined as the subjective symptoms $(0-20){ }^{2}$ Clinical information, diagnosis and SCORAD assessment were conducted by a consulted dermatologist.

\section{Biochemical measurement}

The venous blood samples were taken in the morning hours, between 08:00 and 09:00 am from each patient (atopics who were in exacerbation periods and the controls) at one time. Serum prolactin levels were measured with chemiluminescence (Abbott, USA) method. Normal values were 5.30-63.3 ng/mL for 2-12 months old; $4.40-29.7 \mathrm{ng} / \mathrm{mL}$ for $2-3$ years old; $2.63-21 \mathrm{ng} / \mathrm{mL} 4-11$ years old; $2.84-24 \mathrm{ng} / \mathrm{mL}$ for 14-18 years old males and 2.52-16.9 ng/mL for 14-18 years old females. Normal values for older than 18 years old were $2.5-18 \mathrm{ng} / \mathrm{mL}$ for men and $1.2-30 \mathrm{ng} / \mathrm{mL}$ for women.

\section{Statistical analysis}

All data were analyzed by SPSS software 20.0 version using Mann-Whitney U-test and Spearman correlation analysis for statistical evaluation. A $p$ value $\leq 0.05$ was considered significant.

\section{RESULTS}

This study included 46 patients with atopic dermatitis of whom 30 were males and 16 females, aged 0.5 to 19.5 years (mean, $5.95 \pm 4.71$ years) and 100 controls, of whom 60 were males and 40 females, aged 0.16 to 16 years (mean, $6.46 \pm 4.27$ years).

TABLE 1. The clinical features of the patients

\begin{tabular}{ll}
\hline Number of patients with atopic dermatitis/Number of non-atopic controls & $46 / 100$ \\
Age, years (mean \pm SD) & 0.5 to $19.5(5.95 \pm 4.71)$ \\
Sex & 30 male $(65.2 \%)$ \\
Duration of disorder, years (median range) & 16 female $(34.8 \%)$ \\
Number of relapses, per year (median range) & $1(0.08-16.25)$ \\
SCORAD (median range) & $2(1-24)$ \\
\hline
\end{tabular}

SCORAD: SCORing Atopic Dermatitis. 
The median duration of atopic dermatitis was 1 year (range: $0.08-16.25$ years) and the median of the relapses was 2 per year (range: 1-24). The median value of SCORAD was 31 (9-64) (Table 1).

Neither patients nor the controls had hyperprolactinemia. Median (range) serum prolactin concentration was $10.54 \mathrm{ng} / \mathrm{ml}$ (3.9230.24) for atopic dermatitis patients and 11.47 (3.13-48.84) for controls; p: 0.267. No significant difference was found in prolactin levels in both groups ( $p>0.05)$.

No significant correlation was found between prolactin levels and sex, duration, number of relapses and scores of atopic dermatitis $(p>0.05$, Spearman correlation analysis).

\section{DISCUSSION}

In our study, serum levels of prolactin in patients with atopic dermatitis were not higher than non-atopic individuals. Besides, there was no correlation between disease severity and serum levels of PRL. There are few reports about serum levels of PRL in atopic dermatitis. Similar to our study, Handjani et al. reported no significant difference in the serum prolactin levels between patients with psoriasis and atopic dermatitis and the control group. ${ }^{9}$ In the study of Kasperska-Zajac et al., serum concentration of prolactin was measured in 13 female patients with severe intensity of atopic eczema/dermatitis syndrome and compared next with 14 healthy subjects. The study failed to detect any significant differences in serum basal concentration of prolactin among patients and the healthy subjects as our results. ${ }^{10}$ Giasuddin et al. measured serum PRL levels in 12 patients with psoriasis vulgaris, and found that they were significantly higher than those of 9 patients with atopic dermatitis and 20 controls whereas there was no significant difference between atopic patients and controls. ${ }^{7}$

Girolomoni et al., showed that PRL at physiological concentrations has an ability to stimulate and maintain proliferation of keratinocytes which may be relevant in hyperproliferation conditions of the epidermis such as psoriasis and atopic dermatitis. ${ }^{4}$

Epidermal hyperproliferation is an important clinical observation in atopic dermatitis and apart from the involvement in the immunological response, keratinocytes contribute to the pathology of atopic dermatitis by a change in their growth characteristics. ${ }^{8}$ Jensen et al. reported that the epidermal proliferation is 5 -fold increased in lesional skin of atopic dermatitis patients compared with healthy individuals. ${ }^{11}$

There are some reports about PRL levels in psoriasis where hyperproliferation of keratinocytes is the hallmark of the disease similar to atopic dermatitis. ${ }^{7}$ PRL levels have been reported to be higher in psoriasis patients in some studies $^{6,7}$, while some other studies claimed no significant difference. ${ }^{5,12}$

The mechanisms underlying the keratinocyte hyperproliferation which is observed in inflammatory skin diseases such as atopic dermatitis and psoriasis are not fully understood yet, although especially growth factors and cytokines, such as TNF- $\alpha$, IFN- $\gamma$ and IL-1 are involved in the growth of keratinocytes. ${ }^{13}$

According to the studies, PRL is involved in the psoriasis etiopathogenesis. ${ }^{12,14}$ Whereas, PRL may not have a role in the etiopathogenesis of atopic dermatitis. The epidermal hyperplasia in atopic dermatitis and psoriasis might be triggered by different mechanisms. For example, IL-21 and IL-23 were shown to induce epidermal hyperplasia only in psoriasis. ${ }^{13}$ Histamine can increase the proliferation of human keratinocytes from atopic dermatitis patients differently from psoriasis. $^{8}$

In a study by El-Khateeb et al., PRL levels were found significantly higher in lesional skin than in non-lesional psoriasis skin and serum. Authors reported that the significant increase in prolactin level in lesional skin compared with serum in psoriasis had addressed assumption of a local increase in prolactin production in lesional skin in psoriasis. ${ }^{15}$ The lack of difference in PRL levels in our and previous studies, might be due to the local production of PRL in atopic lesions similar to psoriasis.

One limitation to our study was that levels of prolactin were measured only once and only in exacerbation periods. Patients with atopic dermatitis frequently admit to the outpatient clinic when they are not in remission period. Another limitation was the smaller sample size comparing non-atopic individuals. The small sample size may be another factor for lack of difference in PRL levels.

As a conclusion, our results do not support the increased secretion of prolactin in $\mathrm{AD}$ and the relationship between the immune-inflammatory processes associated with AD. However, future studies with larger sample sizes might measure PRL levels in the skin and determine possible association with disease severity. 


\section{REFERENCES}

1. Leung DY, Boguniewicz M, Howell MD et al. New insights into atopic dermatitis. J Clin Invest 2004;113(5):651-7.

2. European Task Force on Atopic Dermatitis. Severity scoring of atopic dermatitis: the SCORAD index. Dermatology 1993;186(1):23-31.

3. Paus R. Does prolactin play a role in skin biology and pathology? Med Hypotheses 1991;36(1):33-42.

4. Girolomoni G, Philips JT, Bergstresser PR. Prolactin stimulates proliferation of cultured human keratinocytes. J Invest Dermatol 1993;101(3):275-9.

5. Gorpelioglu C, Gungor E, Alli N. Is prolactin involved in etiopathogenesis of psoriasis? J Eur Acad Dermatol Venereol 2008;22(9):1135-6.

6. Dilmé-Carreras E, Martín-Ezquerra G, Sánchez-Regaña M, et al. Serum prolactin levels in psoriasis and correlation with cutaneous disease activity. Clin Exp Dermatol 2011; 36(1):29-32.

7. Giasuddin AS, El-Sherif AI, El-Ojali SI. Prolactin: does it have a role in the pathogenesis of psoriasis? Dermatology 1992;197(2):119-22.

8. Glatzer F, Gschwandtner M, Ehling S, et al. Histamine induces proliferation in keratinocytes from atopic dermatitis patients. I Allergy Clin Immunol 2013;132(6):1358-67.

9. Handjani F, Saki N, Ahrari I, et al. Serum prolactin levels in psoriasis vulgaris. ISRN Dermatology 2014;2014:586049.

10. Kasperska-Zajac A, Brzoza Z, Rogala B. Serum prolactin concentration in patients suffering from severe atopic eczema/dermatitis syndrome. Centr Eur J Immunol 2008;33(2):54-5.

11. Jensen JM, Fölster-Holst R, Baranowsky A, et al. Impaired sphingomyelinase activity and epidermal differentiation in atopic dermatitis. J Invest Dermatol 2004;122(6):1423-31.

12. Robati RM, Toossi P, Rahmati-RoodsariM, etal. Association of Psoriasis Severity with Serum Prolactin, Thyroid Hormones, and Cortisol before and after Treatment. Scientific World Journal 2013:921819.

13. Keen MA, Hassan I. Serum Prolactin Levels in Psoriasis and its Association with Disease Activity: A Case-Control Study. Indian J Dermatol 2014;59(6):562-6.

14. Roman II, Constantin AM, Marina ME, et al. The role of hormones in the pathogenesis of psoriasis vulgaris. Clujul Med 2016;89(1):11-8.

15. El-Khateeb EA, Zuel-Fakkar NM, Eid SM, et al. Prolactin level is significantly elevated in lesional skin of patients with psoriasis. Int J Dermatol 2011;50(6):693-6. 